\title{
MODELING THE EFFECTS OF BEAM SIZE AND FLAW MORPHOLOGY ON ULTRASONIC PULSE/ECHO SIZING OF DELAMINATIONS IN CARBON COMPOSITES
}

\author{
Frank J. Margetan ${ }^{1}$, Cara A. Leckey ${ }^{2}$ and Dan Barnard ${ }^{1}$ \\ ${ }^{1}$ Center for Nondestructive Evaluation, Iowa State University, Ames, IA 50011 \\ ${ }^{2}$ NASA Langley Research Center, Hampton, VA 23681
}

\begin{abstract}
The size and shape of a delamination in a multi-layered structure can be estimated in various ways from an ultrasonic pulse/echo image. For example the -6dB contours of measured response provide one simple estimate of the boundary. More sophisticated approaches can be imagined where one adjusts the proposed boundary to bring measured and predicted UT images into optimal agreement. Such approaches require suitable models of the inspection process. In this paper we explore issues pertaining to model-based size estimation for delaminations in carbon fiber reinforced laminates. In particular we consider the influence on sizing when the delamination is non-planar or partially transmitting in certain regions. Two models for predicting broadband sonic time-domain responses are considered: (1) a fast "simple" model using paraxial beam expansions and Kirchhoff and phase-screen approximations; and (2) the more exact (but computationally intensive) 3D elastodynamic finite integration technique (EFIT). Model-to-model and model-to experiment comparisons are made for delaminations in uniaxial composite plates, and the simple model is then used to critique the $-6 \mathrm{~dB}$ rule for delamination sizing.
\end{abstract}

Keywords: Carbon Composite Laminates, Delaminations, Ultrasonic Pulse/Echo Inspections PACS: 43.20.Ye, 43.20.Fn, 43.60.Lq.

\section{INTRODUCTION AND BACKGROUND}

Suppose that one is performing an ultrasonic pulse/echo inspection of a carbon composite laminate with the aim of detecting delaminations between plies. Some particular inspection setup will be used, perhaps one that involves full waveform capture as the transducer is scanned over the component. For that inspection setup several questions arise. For a given ultrasonic indication what is the best way to analyze the data to estimate the size and shape of the underlying delamination? What is the uncertainty in the sizing estimate? If a component is found to have a small delamination, returned to service, and later rescanned, what is the smallest change in delamination size one can reliably discern? Such questions motivate the present paper. One common approach to delamination sizing, illustrated in Figure $1 \mathrm{~b}$, uses the so called " $50 \%$ " or "- $6 \mathrm{~dB}$ rule". One locates the pixels within an ultrasonic C-scan image for which the measured response is at least $50 \%$ of its maximum value. The boundary of this region then serves as an estimate of the boundary of the delamination. As discussed by R. A. Smith et al. [1] this approach is expected to be quite accurate in some circumstances such as near straight boundaries when the delamination size is larger than the sound pulse spot size, and 


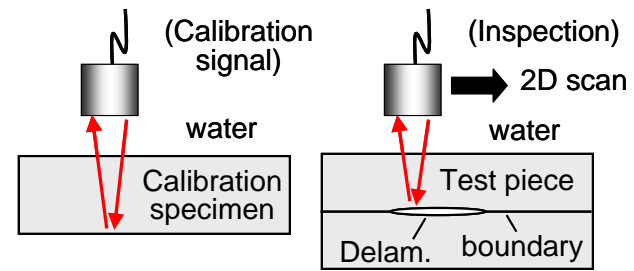

(b)

(c)
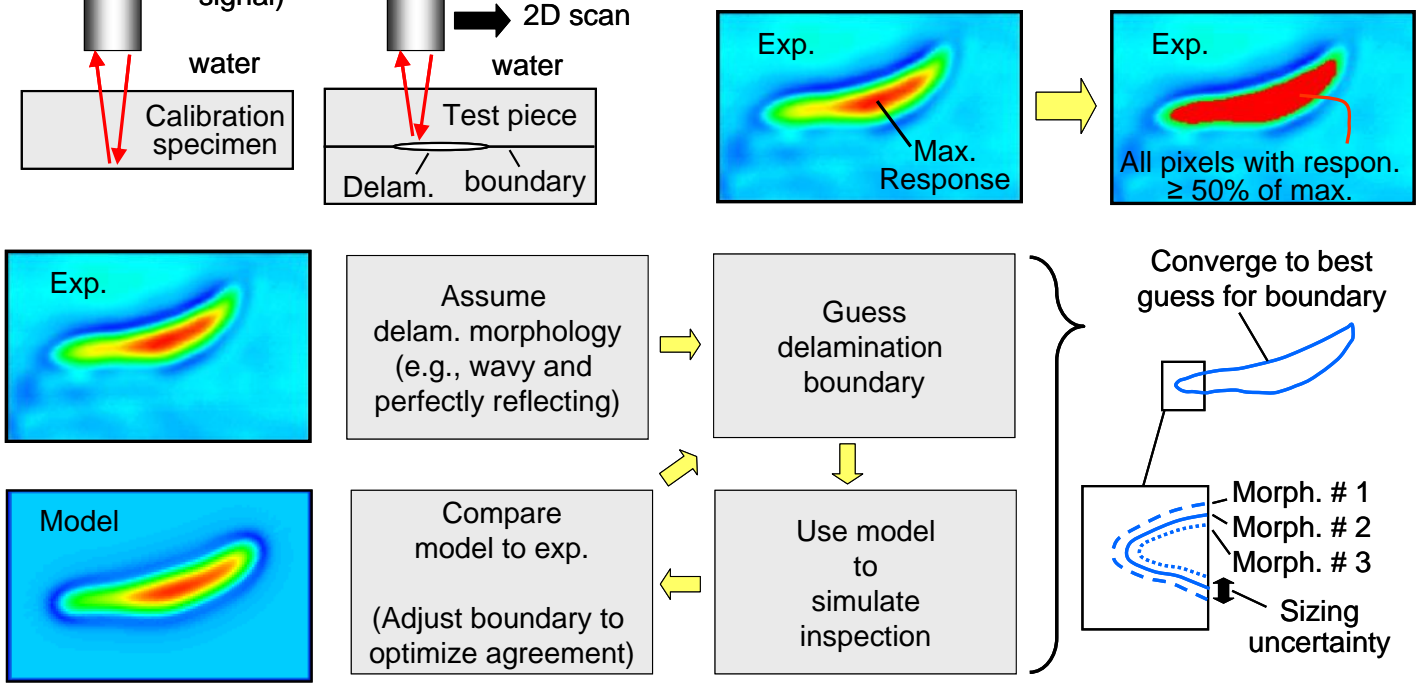

Figure 1. (a) Typical UT inspection setup. (b) Use of the $-6 \mathrm{~dB}$ rule to estimate delamination size (red region) from an amplitude C-scan. (c) An alternative model-based procedure for delamination sizing.

the delamination is flat and has nearly uniform reflectivity. The $-6 \mathrm{~dB}$ rule tends to underestimate the delamination size for convexly curved edges, with the errors becoming larger as the "beam footprint" widens. Such errors can be partially rectified by making "diffraction corrections", and correction rules have been developed for simple delamination shapes [1].

As a possible improvement on the $-6 \mathrm{~dB}$ rule, we have in mind the generic approach shown in Figure 1c where model simulations now play a key role. In addition to the standard UT scan over the delamination, a reference signal is measured, for example a back-wall echo from a calibration block. This signal becomes one model input and serves to capture the effective spectral content of the sound pulse. In more formal language, it is used to determine the efficiency of the measurement system for converting electrical energy into sound and vice versa. Beginning with an estimated delamination boundary, perhaps from the $-6 \mathrm{~dB}$ rule, one uses the model to simulate the inspection. Model and experiment are compared and the model delamination boundary is adjusted to maximize the agreement. In this process one makes choices about the morphology of the model delamination. For example one may begin by assuming a perfectly flat and perfectly reflecting delamination. This leads to one boundary estimate. A second modeling trial may assume a non-planar delamination described by a certain pattern of height variations across its surface, perhaps based on experimental measurements of waviness on documented defects. This leads to a second boundary estimate. This process can be continued for a range of probable morphologies, resulting in a range of estimated boundaries, as indicated in Figure 1c. From that range of estimated boundaries one could arrive at both a best estimate of the delamination size and an estimate of the sizing uncertainty.

For such an approach to be practical one needs an approximate model which takes into account salient physical phenomena (diffraction, attenuation, anisotropy, delamination morphology), but yet is computationally efficient so that $\mathrm{C}$-scans can be rapidly predicted for any given delamination shape and morphology. In this paper we explore the use of one such approximate model based on earlier work by Minachi et al. [2], denoted here as the "simple" model. Its practical accuracy is gauged by comparisons to more exact model calculations using the elastodynamic finite integration technique (EFIT) [3]. We begin by describing several composite specimens that were fabricated to contain real or artificial delaminations. UT measurements on real delaminations are used to demonstrate the need to account for nonplanarity ("waviness") of the delamination surface. The two models are then briefly described and applied to several delaminations with similar dimensions but different surface morphologies. Measured C-scans for the artificial-delamination specimens are then compared 
with the predictions of the simple model. Finally, the simple model is used to study the influence of waviness and partial transmissivity on delamination sizing, and to estimate typical sizing errors when the $-6 \mathrm{~dB}$ rule is applied to one particular inspection.

\section{COMPOSITE SPECIMENS AND INSPECTION SETUPS}

Flat test plates were constructed by stacking 26 Layers of uniaxial carbon/epoxy prepreg material (IM7/8552) in either a uniaxial or quasi-isotropic layup. For some of the specimens artificial delaminations $(0.25$ " $\mathrm{x} 0.25$ " squares by design) were created by introducing doublelayers of thin Teflon tape between layers 13 and 14. After curing, plate dimensions were approximately 6.0 ” 2.6 " 0.13 ”. In this paper we discuss only the uniaxial plates. For this group, elastic stiffness constants were determined from measurements of longitudinal and shear sound speeds for various propagation directions. Longitudinal-wave attenuation in the throughthickness direction was then determined by comparing the spectral amplitudes of successive back-wall echoes using a $10-\mathrm{MHz}$ broadband transducer at normal incidence. Plate properties are summarized in Figure $2 \mathrm{~b}$ and serve as inputs to the simulation models we discuss later. As an alternative to the artificial delaminations, real delaminations could be induced by notching one edge of a plate and inserting a wedge, as shown in Figure 2d.

The two inspection setups used throughout this paper are shown in Figure 2a. Each involves a 2D raster scan of a broadband transducer above a delamination. Setup 1 used a 10$\mathrm{MHz}, 0.75$-inch diameter, $\mathrm{F}=1$ ", focused transducer with the water path chosen to focus at the specimen mid-plane. This was used to look at the delamination structure itself, i.e., the waviness of the surface, and any variations in local reflectivity. Setup 2 used a $10-\mathrm{MHz}, 0.25$ ”-

(a)

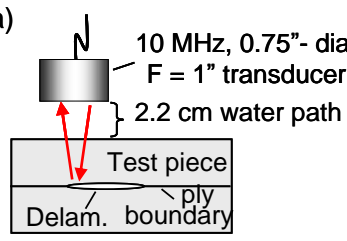

Setup 1

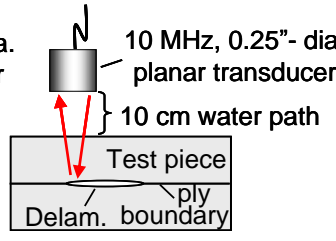

Setup 2

(c) Typical A-scan:

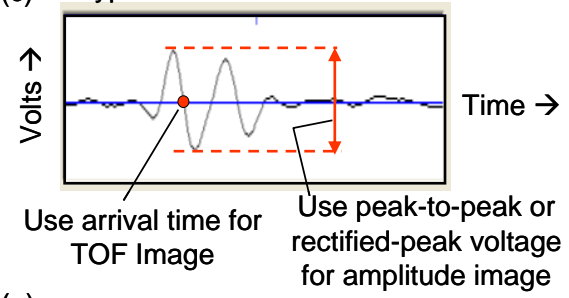

(e)

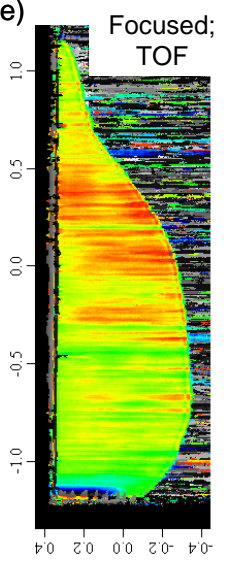

(b)

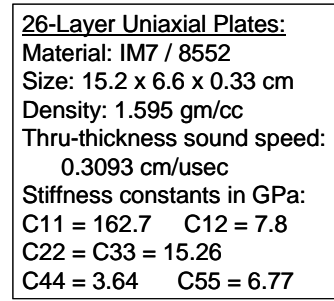

(d)

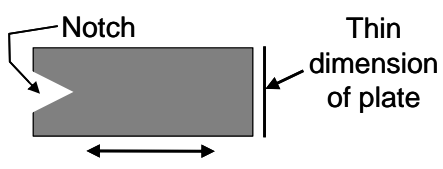

Fiber direction
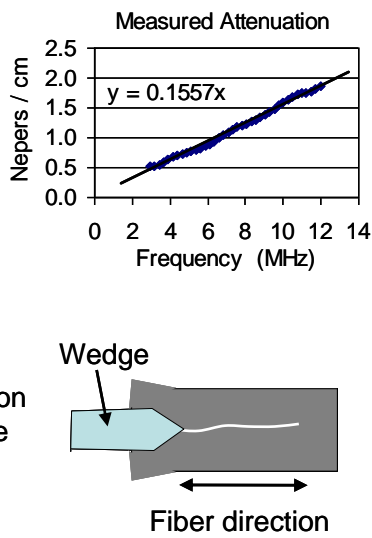
for amplitude image

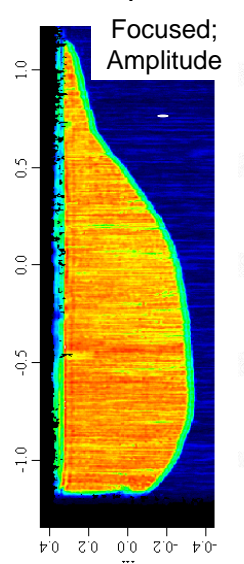

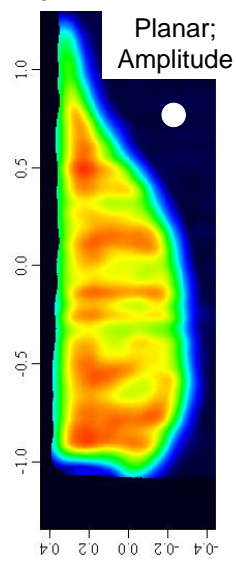

(f)

Focused Transducer; Typical B-scans along 0.6 inch lengths

Across Fibers Parallel to Fibers
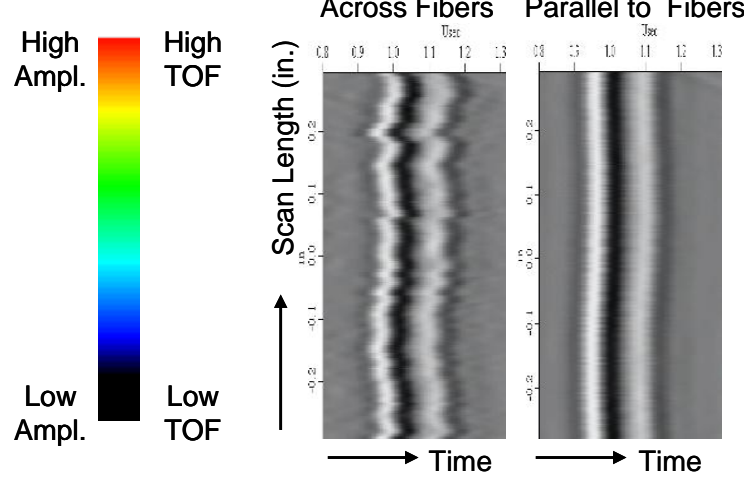

Figure 2. (a) Inspection setups 1 and 2. (b) Measured properties of the uniaxial composite plates. (c) Waveform attributes used in C-scans. (d) Method for inducing a real delamination. (e) Amplitude and TOF C-scans of a real delamination using the focused and planar transducersetups. (Carbon fibers run horizontally.) (f) Typical B-scans for focused transducer movement across the delamination. 
(a)

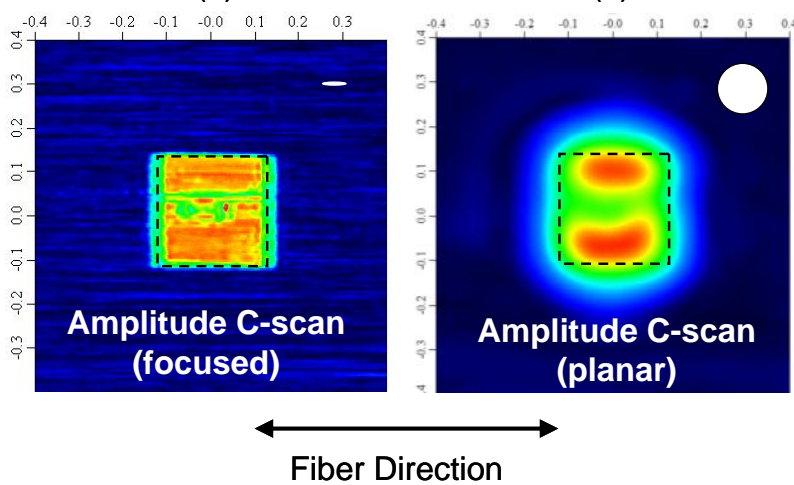

(c)

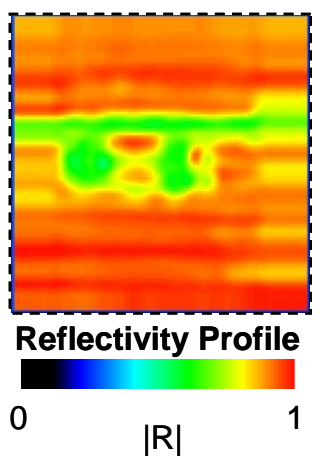

(d)

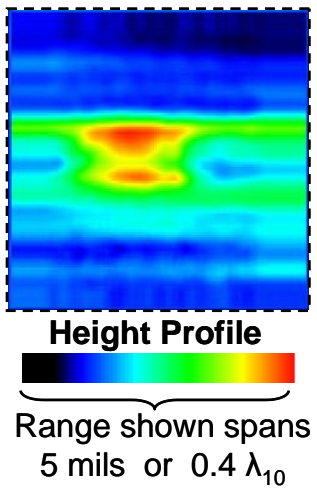

Figure 3. Amplitude C-scans for artificial delamination $8 \mathrm{U}$ using the focused (a) and planar (b) transducer setups. The scan area is 0.8 " $\mathrm{x} 0.8$ " and the white region indicates the approximate size of the sound-pulse footprint at the delamination depth. Assumed reflectivity (c) and height (d) profiles for a 0.25 " x 0.25 " model delamination which emulates 8U. Dashed black lines in all images are 0.25 " squares.

diameter planar transducer, with a 10-cm water path to place the specimen's mid-plane in the transducer's far field. This was used to study the interesting and challenging case where beam size and delamination size are more comparable. All of our modeling results are for Setup 2.

Inspection results for one real delamination measuring about $2.5^{\prime \prime}$ by 0.7 " are shown in Figure 2e-f. For the focused-transducer inspection horizontal streaking is seen in both the amplitude and time-of-flight (TOF) C-scan images. That streaking is parallel to the fiber direction and is a consequence of waviness (height variations) in the delamination surface. "Crests" of the height variations run parallel to the fiber direction. That variability can also be seen in the B-scan of arrival time versus position for a line scan across the fiber direction. Analysis of the focused-transducer TOF data indicated that the height variations over the delamination surface spanned about $80 \%$ of a ply thickness or about $30 \%$ of the wavelength at the $10-\mathrm{MHz}$ center frequency. Figure $2 \mathrm{e}$ also shows the amplitude C-scan for the planar transducer inspection. There substantial destructive interference can occur as the larger beam footprint simultaneously insonifies peaks and valleys on the delamination surface. The result is a "blotchy" image whose peak amplitude is well below that expected for a flat, perfectly reflecting delamination. In Figure $2 \mathrm{e}$, a white ring or disc indicates the size of the incident sound-pulse footprint at the delamination depth, namely the full width at half maximum amplitude of the incident pressure squared.

We saw similar behavior for three artificial delaminations we studied, denoted here as 7U, 8U and 9U, respectively. Figure 3a-b displays measured amplitude C-scans for one of them $(8 \mathrm{U}) . \quad T h e$ focused transducer scan revealed a square-like delamination of about the 0.25 " design size. The top and bottom edges are better defined than the left and right edges, because of a broadening of the focal spot along the fiber direction due to velocity anisotropy. Notice the region near the center having reduced reflected amplitude. Through-transmission measurements saw some transmitted sound in this region. For use in our later model studies we defined model delaminations which were exactly $0.25 " \times 0.25 "$ in size and which had variations in local reflectivity and surface height (waviness) which mirrored those seen in the artificial delaminations. For the delamination of Figure 3, the reflectivity and height profiles deduced from the focused transducer inspection are shown in panels (c) and (d). These were based on the focused-transducer amplitude and TOF images, respectively. The local reflectivity was assumed to be unity at the locations having the largest measured backscattered response, and proportionally smaller at locations of reduced response. Assigned values near the edges of the delamination were obtained by extending outward the nearby measured interior values. For delamination $8 \mathrm{U}$ the height variations spanned about 1 ply thickness (5 mils), or about $40 \%$ of the wavelength at the $10-\mathrm{MHz}$ center frequency. The results of Figures 2-3 point to the need to treat both wrinkling and partial transmissivity when modeling $\mathrm{P} / \mathrm{E}$ inspections of delaminations. 
(a)

(b)

Delamination response at frequency $\omega$ is proportional to:

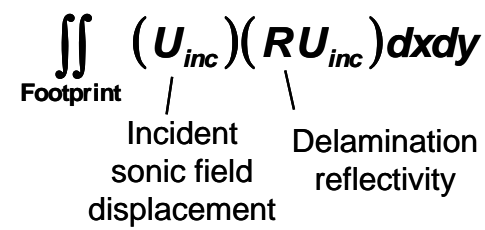

Early time $t_{1}$ : Computed

incident field approaching delam.

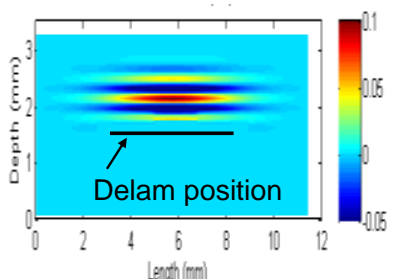

(Through-thickness displacement displayed)

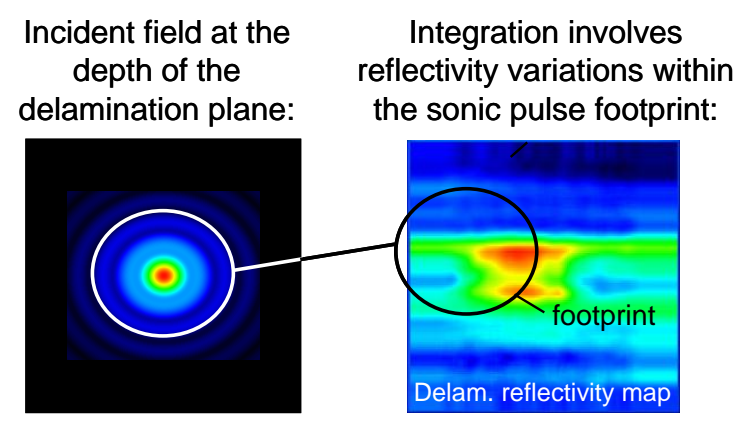

Figure 4. Modeling concepts and details. (a) Height profile assumed in one EFIT model calculation, and resulting 2D through-thickness image slices from the 3D computation. (b) Simple model approach.

\section{MODEL APPROACHES AND COMPARISONS}

The more accurate of the two models used here employed the EFIT approach. Details about its implementation in composite materials may be found in a companion article in these Proceedings [3]. The model volume is first discretized in both space and time, with volume elements being small compared to the sonic wavelength. The physical properties within each volume element are specified, and boundary conditions are used to connect adjacent elements. An initial sonic impulse is specified, and the equations of linear elastodynamics are used to compute the time evolution of the propagating sonic fields. An example is illustrated in Figure 4a. There a particular model delamination is specified, in this case one having a wavy surface profile modeled after delamination $8 \mathrm{U}$. At early time $\mathrm{t} 1$, a broadband sonic pulse is seen approaching the delamination. At later time $\mathrm{t} 2$, the fields scattered by the delamination are seen. Only two-dimensional slices are shown in Figure $4 \mathrm{~b}-\mathrm{c}$, but the calculations are fully three dimensional. To compute the A-scan response for the model delamination, the reflected field is integrated over the surface of a receiving transducer.

The simpler model used here is a modification of that proposed by Minachi [2] for simulating the UT response from a planar delamination. It makes use of a paraxial expansion for the propagation of a finite beam in a homogeneous anisotropic medium, and uses a Kirchhoff approximation for computing the response from the delamination. In computing the delamination response, one essentially integrates the product of the incident and reflected sonic displacement fields over the surface of the delamination, with the reflected field being proportional to the product of the incident field and the local reflection coefficient. We modify Minachi's model in two respects: (1) replacing the Gauss-Hermite beam expansion by the simpler Multi-Gaussian expansion; and (2) introducing a variable reflectivity to deal with typical delamination morphologies. Like Minachi, we keep the surface of the "calculational plane" flat. For the cases at hand (setups 1 and 2), this plane is normal to the transducer's central ray direction, and at the same depth as the average depth of the delamination's surface. On the calculational plane we pre-compute and store incident sonic field quantities for each discrete frequency within the transducer's bandwidth. As indicated in Figure $4 \mathrm{~b}$ the sonic response at a given transducer location is obtained by multiplying the local reflectivity by the 
(a)

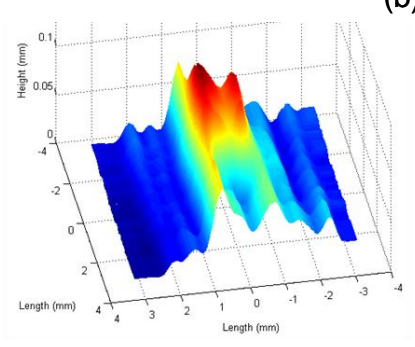

(b)

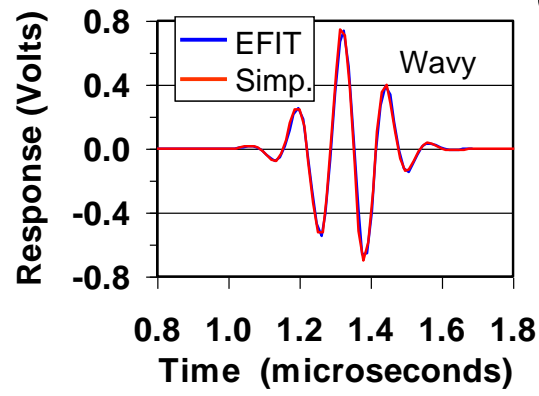

(c)

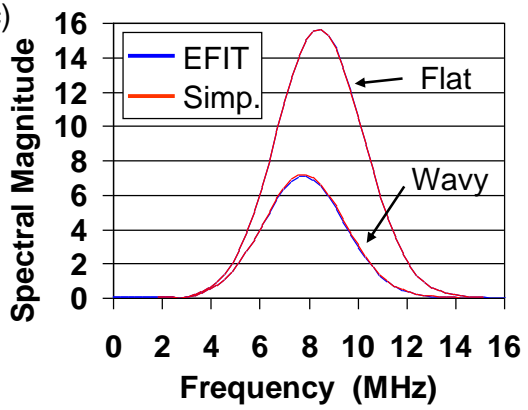

Figure 5. Predicted responses using the EFIT and Simple models for the inspection of a 0.25 " x 0.25 " delamination in a uniaxial composite plate. (a) Assumed delamination height profile (ala 8U). (b) Predicted time-domain responses. (c) Predicted spectral magnitudes for this delamination and a flat one of similar size

stored incident field quantities and integrating over the portion of the calculational plane that contains the projected area of the delamination. Waviness (variation in delamination height) is treated by a phase-screen approximation, i.e., by a frequency-dependent alteration of the phase of the local reflection coefficient. In particular, the applied phase shift is $2 \mathrm{ik} \Delta \mathrm{z}$ where $\mathrm{k}$ is the longitudinal wavenumber in the through-thickness direction for composite material and $\Delta \mathrm{z}$ is the height of the delamination point above the calculational plane. Transmissivity variations are treated by modifying the amplitude of the reflection coefficient. Ultrasonic A-scans are constructed via the inverse Fourier transform of the computed single-frequency responses.

Model-to-model comparisons were made for several delamination morphologies, ranging from flat delaminations to those having considerable waviness. In each case the delamination's projected area was assumed to be a 0.25 " $\mathrm{x} 0.25$ " square, and the simulations roughly emulated an inspection with the $10-\mathrm{MHz}$ planar transducer (setup 2). The predicted response from a very large flat delamination effectively served as a calibration signal, and that assumed response was identical for both models. Good model-to-model agreement was generally found as illustrated for one case in Figure 5. There the assumed delamination waviness profile was that associated with artificial delamination $8 \mathrm{U}$, and the transducer was assumed to be centered directly above the delamination. Predicted A-scans for the backscattered echo from the delamination are seen to be nearly identical for the two model treatments. Figure 5c compares predicted spectral magnitudes for responses from wavy and flat delaminations. Notice how delamination waviness, and the associated opportunity for destructive interference, reduces the response at the higher frequencies.

Having tested the simple model via comparisons to EFIT, we next turned to model-toexperiment comparisons. As mentioned earlier, focused-transducer scans (Setup 1) had been used to infer reflectivity and height profiles for delaminations $7 \mathrm{U}, 8 \mathrm{U}$ and $9 \mathrm{U}$. Those profiles were assigned to model delaminations whose projected sizes were assumed to each be precisely 0.25 " $\mathrm{x} 0.25$ " squares. We then used the simple model to predict C-scans for inspections using measurement Setup 2, i.e., the inspection with the $10-\mathrm{MHz}, 0.25$ "-diameter planar transducer. In doing this we made use of a measured back-wall calibration signal from a fused quartz block that had been acquired at the same time that the delamination specimens were scanned. This allowed us to make absolute model predictions without introducing arbitrary scaling factors. Results are shown in Figure 6a, and good agreement is seen between the measured and predicted amplitude C-scans. Each C-scan in Figure 6 has its color bar individually set such that black corresponds to zero amplitude and red to the highest amplitude appearing in the image. Thus we are effectively only comparing image patterns and not absolute amplitudes. Absolute amplitudes are compared in Figure $6 \mathrm{~b}$, and in each case the predicted peak amplitude was within $2 \%$ of the measure peak amplitude. For our three artificial delaminations, the measured reflectivity and height profiles had similar overall variabilities, e.g., similar values of rms roughness $(0.68+/-0.05$ mils $)$, and similar values of average reflectivity $(\langle|R|\rangle=0.84+/-$ $0.03)$. However, they of course differed in the details of their waviness and reflectivity profiles. Delamination $7 \mathrm{U}$ had the highest roughness, but produced the largest ultrasonic response, 
primarily because it was smoother in the central region where the peak response would occur for a flat delamination, and hence that maximal response was reduced less by the waviness.

A wide range of frequencies contribute to the broadband images shown in Figure 6a. It is instructive to also look at single frequency images made by displaying the spectral magnitude at a particular frequency versus the two transducer scan coodinates. For example Figure 6c shows single-frequency amplitude images at four frequencies from 2.4 to $9.8 \mathrm{MHz}$. Results are shown for three cases: the model result for a 0.25 " $\mathrm{x} 0.25$ " flat delamination, and model and experimental results for artificial delamination $8 \mathrm{U}$. As one goes down in frequency, the soundpulse footprint (indicated by the white circle) becomes larger due to the effect of diffraction acting over the $10 \mathrm{~cm}$ water path, and the inspection is less effective at discerning the delamination shape. The apparent shape is diamond-like at low frequency, rounder at mid frequencies, and becomes slightly more squarish at higher frequencies. Thus varying the frequency here is akin to varying the beam spot size.

\section{IMPLICATIONS FOR DELAMINATION SIZING}

One can take any of the C-scan images shown in Figure 6 and apply the $-6 \mathrm{~dB}$ rule. In Figure 7 we have done that for the single frequency images for four model delaminations, one being flat and the others emulating the three artificial delaminations $7 \mathrm{U}, 8 \mathrm{U}$ and $9 \mathrm{U}$. Each

(a)
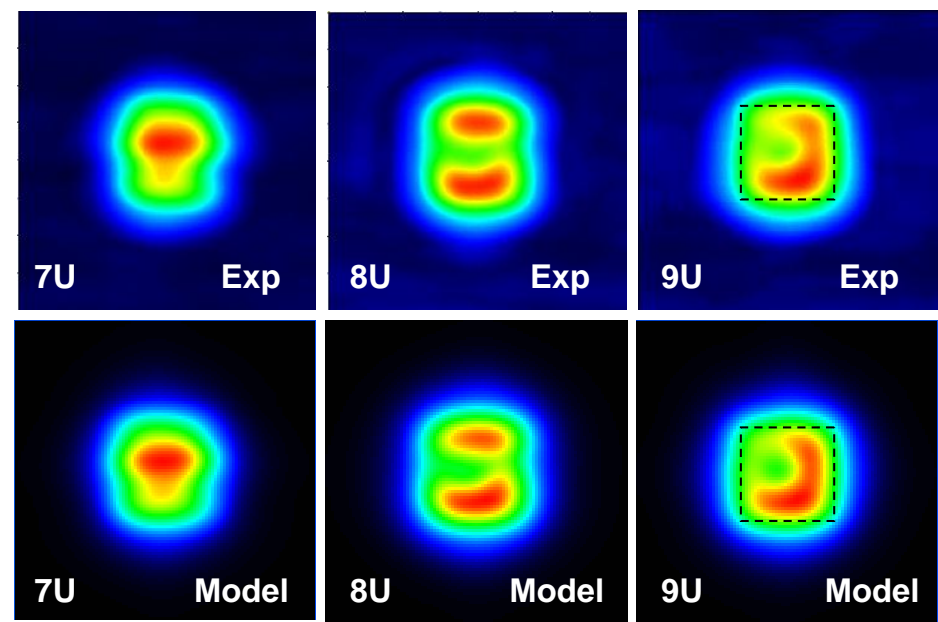

(c)
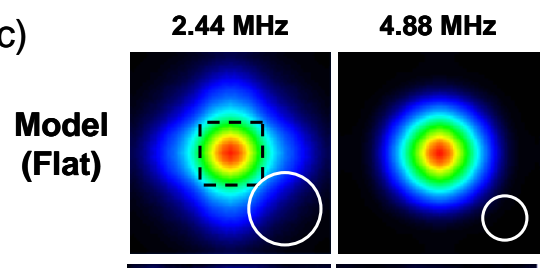

Exp.
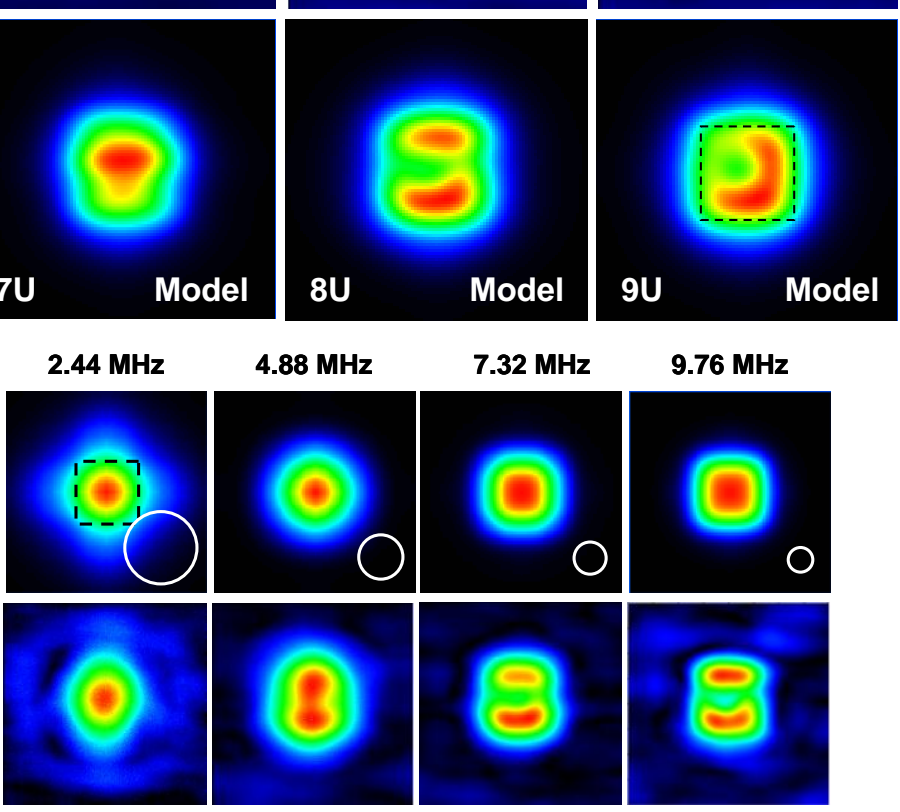$$
\text { (8U) }
$$
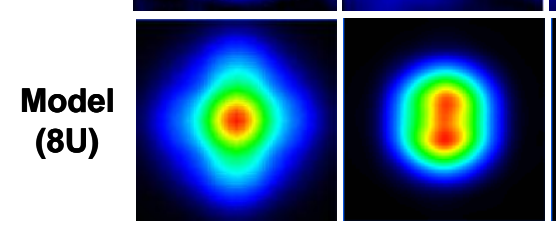

\begin{abstract}
$9.76 \mathrm{MHz}$
\end{abstract}
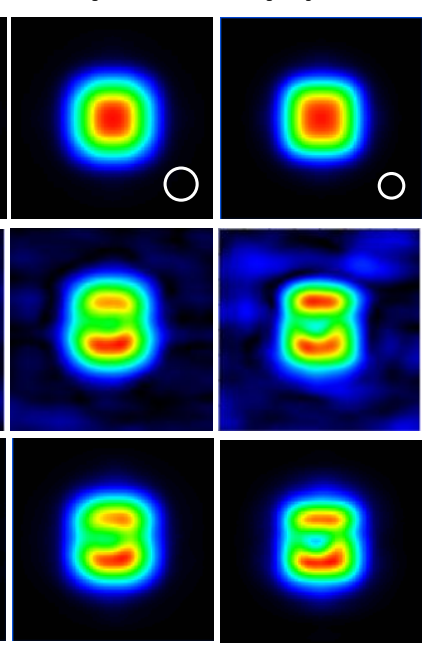

(b)
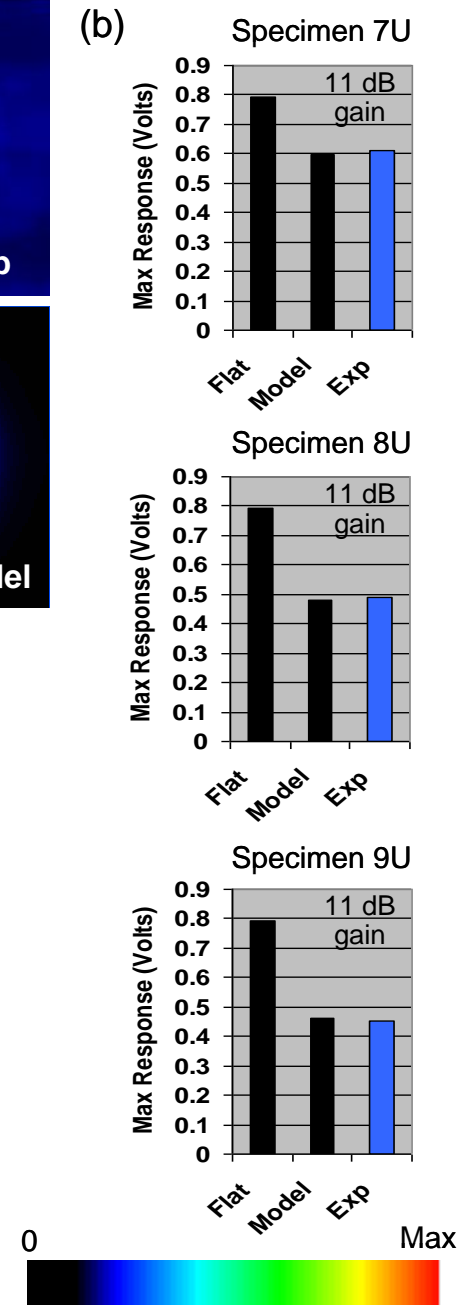

Figure 6. (a) Measured and predicted broadband gated-peak amplitude C-scans for delaminations 7U, 8U, and 9U. (b) Measured and predicted maximal responses for the C-scans of panel (a). Here "Flat" denotes the model results for a flat, perfectly reflecting 0.25 " x 0.25 " delamination. (c). Single frequency C-scan images for delamination $8 \mathrm{U}$, as measured and predicted. Each C-scan shown covers a 0.8 " x 0.8 " area. 
(a)

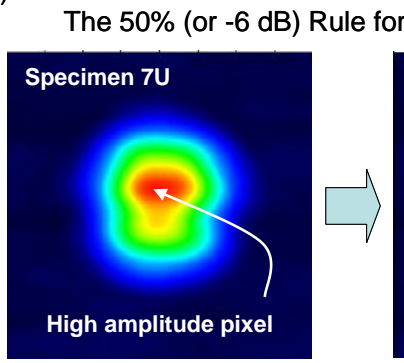

Square with same area has $S=0.232$ inches :

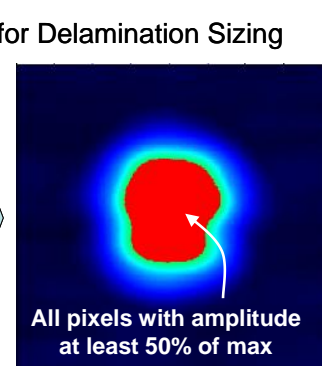

S

$\mathrm{S}$

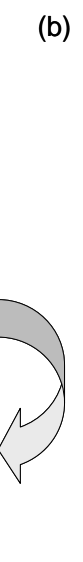

Figure 7. (a) The -6dB sizing rule applied to the broadband C-scan image for artificial delamination $7 \mathrm{U}$. (b) The sizing rule applied frequency-by-frequency to four model delaminations, each of which is a square of side 0.250 " (solid curves). Plotted points result from similar analyses of the measured UT scans for two of the delaminations (7U and $8 \mathrm{U}$ ).

model delamination has a projected area which is square in shape and precisely 0.25 " $\mathrm{x} 0.25$ " in size. In applying the $-6 \mathrm{~dB}$ rule, recall that we first find the maximum amplitude pixel in an image, then locate all pixels having amplitudes within $6 \mathrm{~dB}$ of that value. The combined area (A) of those pixels is our ultrasonic estimate of the delamination area. Since we are dealing with square delaminations here, we go a step further and compute the side of a square $S=A^{1 / 2}$ with that area. In Figure $7 \mathrm{~b}$ we plot that inferred square size versus frequency (or relatedly versus beam spot size). At very low frequencies the beam spot is much larger than the delamination so we are effectively "sizing the beam". Beyond about $2 \mathrm{MHz}$, however, the delamination size comes significantly into play. Since the four model delaminations are 0.25 " squares by definition, any deviation away from $0.25^{\prime \prime}$ in Figure $7 \mathrm{~b}$ is a sizing error. For a flat compact delamination, it is well known that the $-6 \mathrm{~dB}$ rule undersizes the delamination, and we see that here. For more realistic delaminations, however, the sizing error can be either positive or negative depending on the delamination morphology. The vertical spread of the curves in Figure $7 \mathrm{~b}$ supplies a rough estimate of the uncertainty in using the $-6 \mathrm{~dB}$ rule in our circumstance.

The above exercise provides an example of how the simple model can be used to critique a sizing method and to estimate sizing uncertainties. Our studies to date have found the simple model to be both computationally efficient and reasonably accurate. Thus it is a good candidate for pursuing model-based sizing along the lines suggested earlier in Figure 1c. In this endeavor it would very useful to have a database of representative realistic delamination morphologies including information on non-planarity (waviness profiles) and on the scale and patterning of sonic reflectivity (or transmissivity) variations.

\section{ACKNOWLEDGEMENT}

The portion of the work performed at the Center for Nondestructive Evaluation at Iowa State University was supported by NASA under award NNX07AM15A.

\section{REFERENCES}

1. R. A. Smith, A. B. Marriott, and L. D. Jones, "Delamination Sizing in Fibre- Reinforced Plastics using. Pulse-Echo Amplitude.” Insight, Vol. 39, No. 5. May 1997, pp. 330-336.

2. A. Minachi, F.J. Margetan and D.K. Hsu, "Delamination sizing in composite materials using the Gauss-Hermite beam model", Ultrasonics Vol.31, No.4, p. 237 (1993).

3. C. A. C. Leckey, M. D. Rogge, and R. F. Parker, "Microcracking in Composite Laminates: Simulation of the Effects on Ultrasonic Waves", these Proceedings. 